\title{
Video Article \\ The Dig Task: A Simple Scent Discrimination Reveals Deficits Following Frontal Brain Damage
}

\author{
Kris M. Martens ${ }^{1}$, Cole Vonder Haar ${ }^{1}$, Blake A. Hutsell ${ }^{1}$, Michael R. Hoane ${ }^{1}$ \\ ${ }^{1}$ Department of Psychology, Southern Illinois University at Carbondale
}

Correspondence to: Michael R. Hoane at mhoane@siu.edu

URL: https://www.jove.com/video/50033

DOI: doi:10.3791/50033

Keywords: Neuroscience, Issue 71, Medicine, Neurobiology, Anatomy, Physiology, Psychology, Behavior, cognitive assessment, dig task, scent discrimination, olfactory, brain injury, traumatic brain injury, TBI, brain damage, rats, animal model

Date Published: $1 / 4 / 2013$

Citation: Martens, K.M., Vonder Haar, C., Hutsell, B.A., Hoane, M.R. The Dig Task: A Simple Scent Discrimination Reveals Deficits Following Frontal Brain Damage. J. Vis. Exp. (71), e50033, doi:10.3791/50033 (2013).

\section{Abstract}

Cognitive impairment is the most frequent cause of disability in humans following brain damage, yet the behavioral tasks used to assess cognition in rodent models of brain injury is lacking. Borrowing from the operant literature our laboratory utilized a basic scent discrimination paradigm $^{1-4}$ in order to assess deficits in frontally-injured rats. Previously we have briefly described the Dig task and demonstrated that rats with frontal brain damage show severe deficits across multiple tests within the task ${ }^{5}$. Here we present a more detailed protocol for this task. Rats are placed into a chamber and allowed to discriminate between two scented sands, one of which contains a reinforcer. The trial ends after the rat either correctly discriminates (defined as digging in the correct scented sand), incorrectly discriminates, or 30 sec elapses. Rats that correctly discriminate are allowed to recover and consume the reinforcer. Rats that discriminate incorrectly are immediately removed from the chamber. This can continue through a variety of reversals and novel scents. The primary analysis is the accuracy for each scent pairing (cumulative proportion correct for each scent). The general findings from the Dig task suggest that it is a simple experimental preparation that can assess deficits in rats with bilateral frontal cortical damage compared to rats with unilateral parietal damage. The Dig task can also be easily incorporated into an existing cognitive test battery. The use of more tasks such as this one can lead to more accurate testing of frontal function following injury, which may lead to therapeutic options for treatment. All animal use was conducted in accordance with protocols approved by the Institutional Animal Care and Use Committee.

\section{Video Link}

The video component of this article can be found at https://www.jove.com/video/50033/

\section{Introduction}

Brain damage resulting from traumatic brain injury (TBI) and stroke is one of the leading causes of death and disability in the United States. Together, these disorders result in a total of nearly 2.5 million incidences, with nearly 500,000 resulting in death or impairment ${ }^{6-7}$. Despite this heavy toll on society, very few treatments have been developed to treat either TBI or stroke. To some extent, the lack of available treatment options can be attributed to inadequate behavioral assessments of novel drugs and therapies ${ }^{8}$. In particular, there is a lack of tasks to accurately and sensitively evaluate the effects of treatments on the various cognitive deficits following brain injury in the preclinical literature. Currently the majority of cognitive assessments that are performed evaluate spatial memory using a maze task such as the Morris water maze ${ }^{9}$. There are multiple reasons for this lack of testing including the time it takes to fully characterize and evaluate cognitive deficits, the heavy reliance upon expensive equipment and expertise to utilize operant tasks, and the speed at which these evaluations can be performed. To this end, we have adapted a basic scent discrimination task, the Dig task, to assess cognition following brain injury. The Dig task is designed to be a cheap, rapid, and effective tool for evaluating cognitive dysfunction following brain injury from a TBI or stroke.

The Dig task relies on the basic operant principles of learning and decision making. As it is only a two-choice decision paradigm, this task represents one of the simplest discriminations that can be made. In the protocol below the details for setting up the task as well as the training methodology will be described. We have found that this task is strongest when assessing frontally-mediated cognitive deficits compared to unilateral parietal deficits; however, it has yet to be tested under a model of hippocampal damage ${ }^{5}$. Ideally, this task would be suited for evaluating the effectiveness of a treatment or therapy on recovery of frontal deficits following an injury. However, it is possible that with modifications this task could also be used to explore more complex decision-making behavior such as impulsivity and generalized matching.

\section{Materials Required for Training and Testing}

1. Testing chamber. Any container large enough to accommodate one animal and two scent cups. Our laboratory utilized modified operant chambers (Med Associates Inc.) with floor bars removed to make space for scent cups (Figure 1). 
2. Scent cups and cup holder. Cups sufficiently wide and deep to allow for digging and some mechanism/holder to stabilize them (Figure 2).

3. Scented sands. Our testing protocol used scents previously established to not show response bias in rats (cocoa, basil, cumin, coffee) ${ }^{4}$. Each scented sand was mixed at a ratio of $1 \mathrm{~g}$ odorant to $110 \mathrm{~g}$ unscented, clean playground "sand box" sand and can be stored in airtight containers for up to 6 months.

4. Reinforcer. We utilized fruit loop cereal pieces as a reinforcer. We recommend exposing animals to the reinforcer (via placement in their home cages) 3 to 5 days prior to training to overcome neophobic responses.

5. Stop watch or timer.

\section{Animal Handling Prior to Training}

1. Handling. As animals will be placed in and removed from the chambers repeatedly throughout training/testing, we recommend handling animals three to five days prior to training in order to familiarize them with human contact.

2. Motivating. In order to motivate animals to perform on this task, some level of food deprivation is necessary. Food restriction should be initiated at least three days prior to both training and testing and animals may be maintained at $90 \%$ of free-feeding weight. Typically amounts of 15-20 g of chow per day are sufficient, but animal weights should be checked every other day while food restricting.

\section{Dig Training}

Training an animal to dig in sand can be accomplished in an average of eight sessions over a four day period of time. During dig training, the chamber should be set up by placing two scent cups into the mechanism/holder and placing the scent cup setup into the chamber. The general procedure is detailed below with the goal of shaping the animal from consuming the reinforcer to completely digging in sand to retrieve it.

1. Session 1 - chamber habituation and magazine training. Completely fill the scent cups with the reinforcer (no sand). Place the animal into the apparatus for 30 min allowing the animal to freely consume the reinforcer.

2. Session 2 - sand habituation. Completely fill the scent cups with the reinforcer and pour a small amount of unscented sand into each cup, filling no more than half of the cup. Place the animal into the apparatus for 30 min allowing the animal to freely consume the reinforcer.

3. Session 3 - surface digging. Fill the scent cups with an even mixture of reinforcer and unscented sand, leaving multiple reinforcers exposed. Place the animal into the apparatus for $30 \mathrm{~min}$ allowing the animal to freely consume the reinforcer. In the process of consuming the exposed reinforcers, the animal should uncover additional reinforcers below the surface of the sand. The animal should shift from using its mouth to using its forepaws to remove each reinforcer from the sand.

4. Session 4 - digging. Fill the scent cups with a mixture of 1 part reinforcer to 3 parts unscented sand, leaving 1-2 reinforcers exposed. Place the animal into the chamber for $30 \mathrm{~min}$ allowing the animal to freely consume the reinforcer. The animal should begin to dig with its forepaws to uncover additional reinforcers and remove them from the sand. Animals that do not begin to uncover the reinforcer after a 10 min period of time should be assisted (the researcher should uncover one reinforcer at a time until the animal learns).

5. Session 5 - full digging. Fill the scent cups with a mixture of 1 part reinforcer to 3 parts unscented sand, leaving no reinforcers exposed. Place the animal into the apparatus for $30 \mathrm{~min}$ allowing the animal to freely consume the reinforcer. Animals should be assisted as needed. Some animals may need multiple instances of this session in order to demonstrate digging behavior.

6. Session 6 - trial structure habituation. Place three reinforcers in deep, middle, and shallow positions in the sand in each scent cup. Place the animal into the chamber until all three are retrieved from each cup, assisting as needed. Once all have been retrieved, place the animal into a holding apparatus for 15-30 sec and reset the scent cups as described in this step. Then, place the animal back into the chamber and repeat the process until the animal has completed four total trials. The animal should become habituated to being placed in the holding apparatus and start digging immediately when placed into the chamber. The animal should also demonstrate full digging behavior and retrieve the reinforcer at the bottom of the scent cup.

7. Session 7 - single reinforcers. Place a single reinforcer at half of the depth of the sand in each cup. Place the animal into the chamber and allow it to retrieve both reinforcers, and then place it into the holding apparatus for $30 \mathrm{sec}$. Repeat this process for four total trials. The animal should begin to dig faster and require minimal assistance from the researcher.

8. Session 8 - mock discrimination testing. Fill cups as described in session 7. Give the animal 30 sec to retrieve both reinforcers. Place the animal into the holding apparatus when both have been retrieved or $30 \mathrm{sec}$ has elapsed. Repeat this for the number of trials that will be used in the testing phase. Animals should learn to dig rapidly when placed back into the chamber.

9. Additional sessions. Depending on initial discrimination testing, the experimenter may wish to perform additional sessions to familiarize the animal with the given scents. Rats will tend to initially exhibit neophobia and prefer the familiar scents previously associated with reinforcement ${ }^{10}$.

\section{Discrimination Testing}

1. The researcher should choose a scent pairing, including which scent will be baited with the reinforcer (correct discrimination) and which will not. Additional decisions, such as number of discriminations and reversals, maximum number of days and trials, and whether to pretest prior to injury will be largely based on experimental question (see additional notes below).

2. Rats can be tested and data recorded by hand by the researcher. The researcher should record for each trial: correct or incorrect choice, latency to initiate digging, and trials in which a rat times out on.

a. The number of trials administered should be at the discretion of the researcher (see additional notes below).

b. The length of the trial should be at the discretion of the researcher (see additional notes below).

c. The researcher should randomize the trial structure (left-right location of the scent cups), with the caveat that no more than three trials in a row are on a given side. This will help reduce side bias.

3. In order to initiate each trial, place the animal into the chamber facing the rear, equidistant from each cup. Placing an animal closer to one cup may increase the chance of developing a bias. 
4. If the animal correctly discriminates, allow it to retrieve and consume the reinforcer, and then remove it to the holding apparatus for 30 sec. If it times out or chooses incorrectly, remove it immediately, before it "corrects" itself, and place it into the holding apparatus for 30 sec.

5. Repeat this process for the chosen number of trials.

6. Once an animal achieves a pre-determined criterion (i.e. $>80 \%$ accuracy per day for three consecutive days), the animal may move on to the next discrimination/reversal and repeat the above process.

\section{Additional Notes}

1. Testing in our laboratory found that training and testing work best using one-half of a fruit loop cereal piece to avoid satiation.

2. During training, some animals may need more help than others. We recommend doing what is necessary to achieve digging behavior. However, once testing starts, we never attempt to intervene and improve a rat's digging behavior.

3. Varying the total number of trials may be sufficient for different labs. Testing in our laboratory have used 6,8 , and 12 trials per session, 1 session per day, and have found a preference for the 8 trial setup.

4. Varying the length of the trial may be necessary in some experimental setups (e.g., animals under a stress paradigm may need increased trial times or fewer total trials).

5. A preset criterion should be used in order to determine when an animal has mastered a discrimination. Testing in our laboratory have required an accuracy of greater than $80 \%$ ( $5 / 6$ trials, $7 / 8$ trials, or $10 / 12$ trials) per day for 3 consecutive days. However, a higher criterion ( $85 \%)$ could also be used.

6. Preset thresholds/maximums should be used for the maximum number of days spent on a given scent. This will be largely dictated by research question. For example, our laboratory studies TBI and, as of result of the progressive nature of TBI, rats that have not hit criterion are moved on to a novel discrimination/reversal after 12 days of testing.

7. Deciding whether to pretest or just posttest is a question that is largely determined by experimental direction. Pretesting allows one to perform a memory assessment on the animal post injury, which may be relevant depending on the injury type. However, post testing allows one to examine differences in acquisition rate on the discriminations between groups. Our laboratory has and will continue to use both.

\section{Representative Results}

There are two main variables that can be recorded in the Dig task: Correct/Incorrect and Latency. However, non-responses can also be recorded and analyzed as they may be important when considering motivation. These can be used to generate measures for analysis such as accuracy (\# correct/total trials), response bias ${ }^{11}$, and latency. These measures can be used to assess several different types of learning, depending upon the design of the experiment (see additional notes above). Pretesting of animals allows a researcher to evaluate memory for a learned discrimination. Reversals of learned discriminations allow a researcher to see how well an animal can adapt to a changing contingency. Learning of novel discriminations allows researchers to compare learning across different scent pairings as well as evaluate capacity for novel learning.

Depending upon the design of the study (see additional notes above), these measures can be analyzed in a repeated measures design or can be collapsed into a single variable. Previously, we collapsed accuracy into a measure of cumulative proportion correct in order to analyze each scent discrimination separately ${ }^{5}$. This occurred by summing the number of correct discriminations and dividing by the total number of trials for each discrimination. A series of one-way between-subjects analysis of variances (ANOVAs) were then used to examine group differences for each post-injury scent discrimination. However, more power may be obtained for analysis by utilizing advanced statistical techniques such as mixed effects modeling. One other alternative is to utilize signal-detection theory for data analysis. This is a popular approach in the human decision-making literature and allows the researcher to examine most of the data from a given subject in a single graph and quickly assess the degree of accuracy, bias, and deficits ${ }^{12}$. Choice of analytic approach will be largely driven by experimental question, whether one wants to compare across types of learning, evaluate one single type of learning, or apply a decision-making approach to the data.

Following bilateral frontal brain injury, our laboratory has found that rats suffer large deficits under virtually all types of learning in this task. Frontally-injured animals showed a deficit in memory for a previously learned discrimination as well as difficulty learning the reversal of that scent pairing (Figure 3). Frontal animals also had difficulty learning novel discriminations (Figure 3). In accordance with previous research ${ }^{13}$, there was also a large increase in variability in the frontally injured animals (Figure 3).

In contrast, unilateral parietal injuries in our laboratory have shown no deficits across all the discriminations in this task. Unilateral rats showed no deficits in memory for a previously learned discrimination and also acquired the reversal discrimination very rapidly (Figure 4). They learned novel scent pairings very rapidly as well (Figure 4). In addition, the variability within the unilaterally-injured group was very low, with most rats performing optimally on the task. 


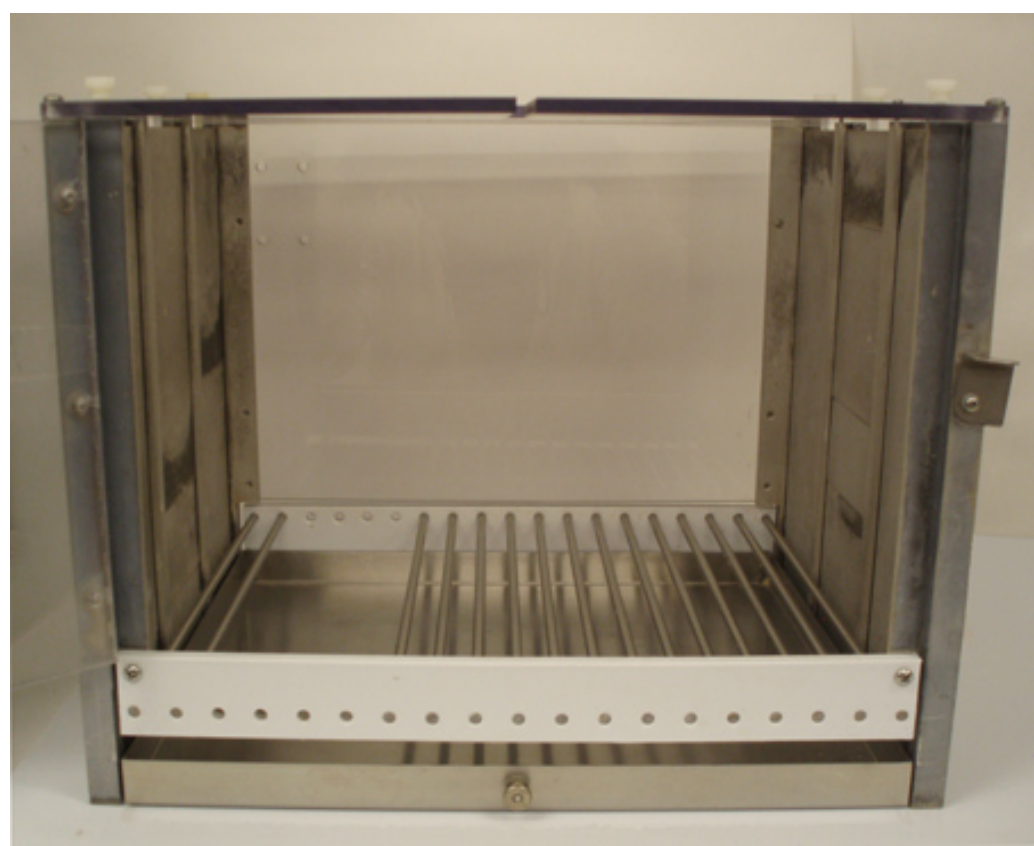

Figure 1. Testing in our laboratory was conducted in a bank of standard operant chambers (Med Associates Inc.) that were adapted for use in this task by removing four consecutive floor-bars to open up space for two scent cups.

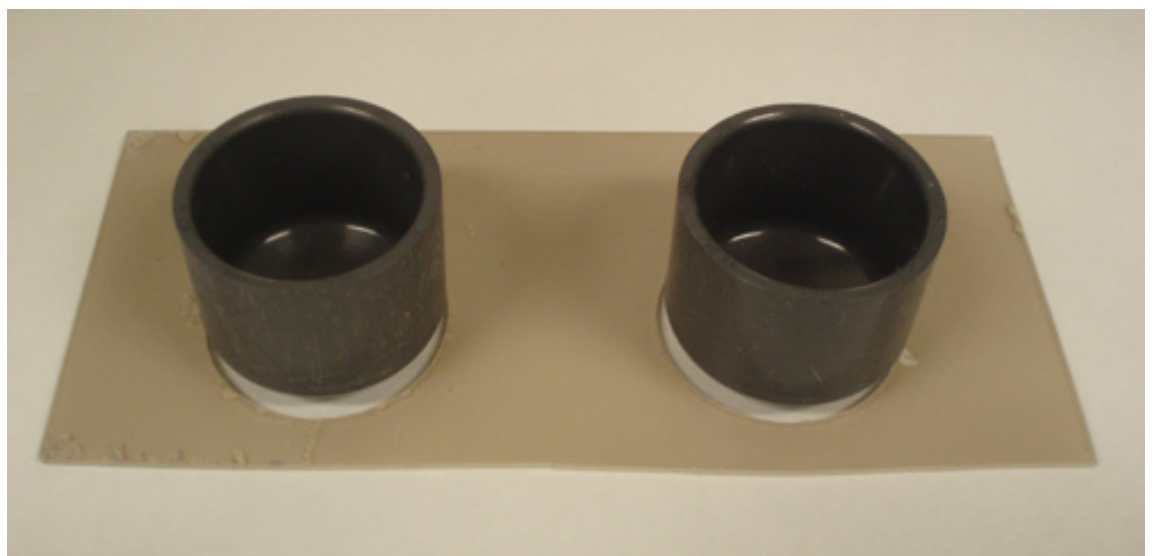

Figure 2. Testing in our laboratory utilized PVC pipe end-caps $(6 \mathrm{~cm}$ tall and $5.5 \mathrm{~cm}$ in diameter) as the scent cups and a piece of Plexiglas (10 $\times 22 \mathrm{~cm}$ ) with two drilled circular holes $(5.5 \mathrm{~cm}$ in diameter) as the scent cup holder. 


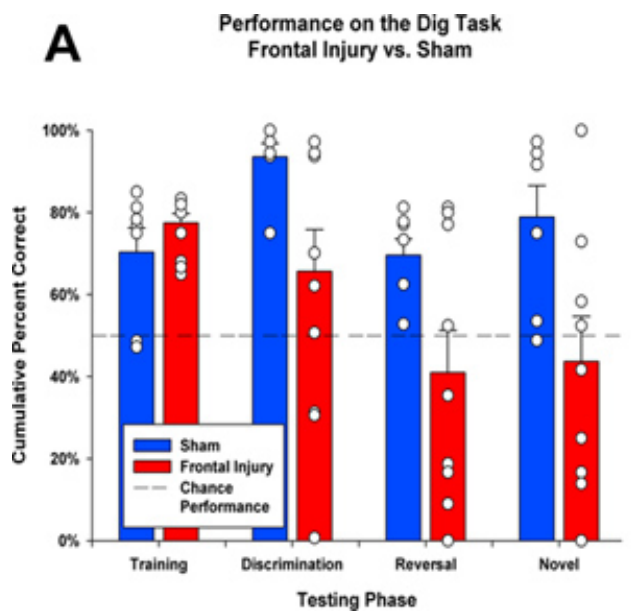
B
Performance on the Dig Task
Frontal Injury vs. Sham

Figure 3. This graph shows typical performance of sham (blue) vs. frontally-injured (red) rats across several different phases of testing in two different formats. In panel A, the data is represented as a bar graph with white dots representing data points for individual rats in each group. In panel $\mathrm{B}$, the same data is graphed in a line graph. There was no difference on the pre-injury discrimination acquisition. However, following injury and on the same discrimination, sham rats perform at $93 \%$ cumulative percent correct while injured rats perform at $65 \%$ cumulative percent correct. When a reversal of the discrimination was administered, injured rats drop to below chance performance. Similarly, on a novel scent discrimination frontally-injured rats had difficulty learning the novel pairing and stayed at below chance performance. Click here to view larger figure.
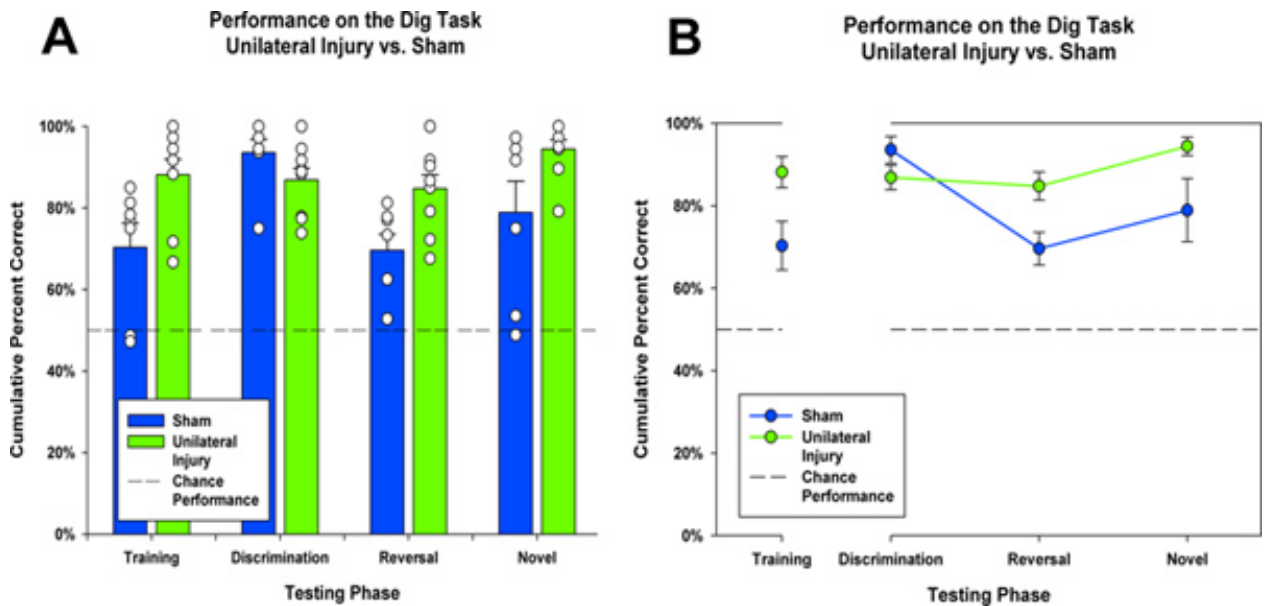

Figure 4. This graph shows typical performance of sham (blue) vs. unilateral parietally-injured rats (green) across several different phases of testing in two different formats. In panel A, the data is represented as a bar graph with white dots representing data points for individual rats in each group. In panel B, the same data is graphed in a line graph. There is no difference between the groups on any of the discriminations. Unilaterally-injured rats performed at or, in some cases, slightly higher than sham levels following injury. Click here to view larger figure.

\section{Discussion}

In this video, we have shown how the Dig task can be conducted using relatively low-cost materials and, with some experience, testing can be conducted fairly rapidly $(\sim 10 \mathrm{~min} / \mathrm{animal})$. This allows for the task to be easily incorporated into existing test batteries for TBI, stroke, and other brain injuries. The Dig task is also robust in that it can assess several different aspects of cognition, including evaluations of previous learning, reversals, and novel acquisitions (e.g., set shifting). Most importantly, the Dig task has been shown to be effective in assessing frontal function following brain damage. In addition, a similar dig paradigm has been used to study value transfer in rats following hippocampal damage ${ }^{4}$, and thus could easily be studied following TBI.

The primary benefits of tasks such as this one are the ability to assess other forms of cognition than what is typically tested in most brain injury studies. There currently exists a lack of simple, rapidly acquired tasks for assessing frontally-mediated cognition following brain damage. In the field of experimental TBI, there is a strong reliance on mazes as the sole assessment of cognition ${ }^{9}$. Discrimination and choice behavior are important aspects of frontally-mediated cognition which need to be evaluated following brain damage. These have been studied under many different paradigms (delayed matching-to-sample, differential reinforcement of low rate of responding, effort-based decision making, etc) in the field of the experimental analysis of behavior, but are lacking in studies of brain injury ${ }^{14-16}$. The development of novel therapeutics for the treatment of brain damage requires validation across multiple modalities of sensory, motor, and cognitive function. Tasks such as this could become vital to fully assessing cognitive function under therapeutic treatment.

While the Dig task represents a step in the right direction for assessing brain function after injury, there are still limitations to it. It is intended to be simple to construct, administer, and analyze, but this means that there are upper limits to what it can measure. Currently, two choices are all that 
can be assessed under the given paradigm. Additionally, the Dig task is not sensitive enough to handle other aspects of frontal dysfunction, such as impulsivity, that may interfere with an animal's decision-making ability.

While the Dig task has been shown to be successful for us in evaluating brain damage in young animals, there are still several things that can be done with the task to further refine and improve it. These include assessing special populations (age, sex, strain), comparing it directly to the operant paradigms it is adapted from, performing a direct comparison of this task with other tasks that assess frontal dysfunction following injury, and exploring additional ways to analyze and compare the data generated from the task. This task is designed to address some of the issues in our field by testing frontal function in multiple ways following brain damage. However, one task cannot measure all the cognitive processes that are impacted by a brain injury. Thus, we advocate that people continue to develop and improve upon tasks such as the one described in this protocol to more fully assess functional impairments following brain injuries. In doing so, we can increase the effectiveness of drug and therapy development for brain injury by assessing across multiple measures.

\section{Disclosures}

No conflicts of interest declared.

\section{Acknowledgements}

Funding for this project was provided by ARRA funds from NINDS grant NS045647.

\section{References}

1. Birrell, J.M. \& Brown, V.J. Medial frontal cortex mediates perceptual attentional set shifting in the rat. J. Neurosci. 20, $4320-4324$ (2000).

2. Bunsey, M. \& Eichenbaum, H. Conservation of hippocampal memory function in rats and humans. Nature. 379, 255-257 (1996).

3. Eichenbaum, H., Fagan, A., \& Cohen, N.J. Normal olfactory discrimination learning set and facilitation of reversal learning after medialtemporal damage in rats: Implications for an account of preserved learning abilities in amnesia. J. Neurosci. 6, 1876-1884 (1986).

4. Kaiser, D.H. \& Means, L. Value transfer across odor stimuli using probability of reinforcement in the rat. Behav. Processes. 73, 164-169 (2006).

5. Martens, K.M., Vonder Haar, C., Hutsell, B.A., \& Hoane, M.R. Additional options for behavioral testing in rodent models of traumatic brain injury: A simple discrimination task used as a novel method of testing decision-making behavior. J. Neurotrauma., In press, (2012).

6. Writing Group Members, et al. Executive summary: Heart disease and stroke statistics - 2012 update. Circulation. 125, 188-197, doi:10.1161/ CIR.0b013e3182456d46 (2012).

7. Center for Disease Control. Injury prevention and control: Traumatic brain injury., (2010).

8. Narayan, R.K., et al. Clinical trials in head injury. J. Neurotrauma. 19, 503-557 (2002).

9. Fujimoto, S.T., Longhi, L., Saatman, K.E., \& Mclntosh, T.K. Motor and cognitive function evaluation following experimental traumatic brain injury. Neurosci. Biobehav. Rev. 28, 365-378 (2004)

10. Mitchell, D. Experiments on neophobia in wild and laboratory rats: A reevaluation. J. Comp. Physiol. Psychol. 90, 190-197 (1976).

11. Baum, W.M. On two types of deviation from the matching law: Bias and undermatching. J. Exp. Anal. Behav. 22, 231-242 (1974).

12. Swets, J.A., Dawes, R.M., \& Monahan, J. Psychological science can improve diagnostic decisions. Psychological Science in the Public Interest. 1, 1-26 (2000).

13. Stuss, D.T., Murphy, K.J., Binns, M.A., \& Alexander, M.P. Staying on the job: The frontal lobes control individual performance variability. Brain. 126, 2363-2380 (2003).

14. Burkett, E.E. \& Bunnell, B.N. Septal lesions and the retention of DRL performance in the rat. J. Comp. Physiol. Psychol. 62, 468-471 (1966).

15. Porter, M.C., Burk, J.A., \& Mair, R.G. A comparison of the effects of hippocampal or prefrontal cortical lesions on three versions of delayed non-matching-to-sample based on positional or spatial cues. Behav. Brain Res. 109, 69-81 (2000).

16. Walton, M.E., Bannerman, D.M., Alterescu, K., \& Rushworth, M.F.S. Functional specialization within medial frontal cortex of the anterior cingulate for evaluating effort-related decisions. J. Neurosci. 23, 6475-6479 (2003). 